\title{
DUST IN NEARBY DWARF GALAXIES
}

\author{
K.-H. SCHMIDT ${ }^{1}$ and T. BOLLER ${ }^{2}$ \\ ${ }^{1}$ Astrophysikalisches Institut, An der Sternwarte 16, Potsdam, Germany \\ ${ }^{2}$ Max-Planck-Institut für Extraterrestrische Physik, Karl-Schwarzschild-Strasse 2, \\ Garching, Germany
}

\section{Introduction}

The aim of the present paper is to look for the gas-to-dust ratio in different types of galaxies and for a correlation between this ratio and the metallicity in dwarf galaxies. The recent compilation of data on nearby galaxies by Schmidt \& Boller (1992, hereinafter SB) including HI and dust masses from IRAS data is used to estimate the gas-to-dust ratio in galaxies of a volume-limited sample.

The special interest is due to this ratio in dwarf irregular systems and spiral galaxies. In order to have well-defined samples we confine ourselves to galaxies of types Sab to Scd brighter than $M(B)=-18$ on the one hand, and to systems of types $\operatorname{Sdm}$ to $\operatorname{Im}$ fainter than $M(B)=-18$. Four peculiar galaxies the masses of $\mathrm{HI}$ and dust of which are also given in the compilation of nearby galaxies are included. These objects have been added to the two groups according to their absolute magnitudes.

\section{Gas-to-Dust Ratio}

The revised catalogue of nearby galaxies (Schmidt et al. 1993) contains 22 objects with given HI and dust masses which belong to the above defined group of spirals. Only one object (Maffei 2) that should be a member of the sample is without reliable knowledge of its dust mass. Thus, the sample is almost complete. In contrast to this group, the sample of irregular galaxies with given data on $\mathrm{HI}$ as well as on dust includes 46 objects, that means about 28 per cent of the total number of irregular galaxies in the catalogue. The completeness decreases with decreasing luminosity. A satisfying completeness of about 70 per cent is present for the range $-16>M(B>-18$, only. Therefore, effects of incompleteness have to be discussed.

The distributions of the HI-to-dust ratio for spiral systems and irregular dwarf galaxies are quite different: irregular systems are, on the average, much less dusty than spiral galaxies. The mean quantities for both the samples are different by a factor 12 in agreement with the previous result by Israel (1984). The spread of the distributions of the HI-to-dust ratio is mainly due to observational uncertainty. However, in the case of the distribution for the spiral galaxies there is a tendency that the early-type spiral systems exhibit preferably smaller gas-to-dust ratios than 
late-type objects. Obviously, part of this effect is due to different content of interstellar molecular hydrogen in different spiral subtypes.

Using the results of Young et al. (1989) and Sage (1993) on the mass of molecular hydrogen in nearby galaxies the HI-to-dust ratios are corrected for molecular hydrogen. Now there is no significant difference in the ratio for different subtypes of galaxies.

\section{The Case of the Non-IRAS Dwarf Irregular Galaxies}

As mentioned above, the dust masses from infrared data and HI masses of only 46 out of 165 dwarf irregulars of the catalogue of SB have been available. The IRAS identified dwarf galaxies are mainly at the luminous limit of the sample. Since the metal content of galaxies is related to the absolute magnitude (Lequeux et al. 1979; Skillman et al. 1989) and the dust is built up by heavy elements, a relation between absolute magnitude and gas-to-dust ratio should be expected. Then the observed mean gas-to-dust ratio of the faint irregulars may be only the observable lowvalue part of a distribution of the gas-to-dust ratio the maximum of which is at a large value of $\log \mathrm{M}(\mathrm{HI}) / \mathrm{M}$ (dust). This gas-to-dust ratio at the place of the maximum of the presumed distribution corresponds to such a low dust mass that will not be seen by IRAS as well as the galaxies of the main part of the distribution of the gas-to-dust ratio.

In order to get an idea if this reflection is correct, the expected far-infrared fluxes of the irregular dwarfs are calculated from the observed $\mathrm{HI}$ masses using different assumptions of the HI-to-dust ratio. Comparing the observed distribution of $\log F(I R)$ with those calculated gives a satisfying agreement for a luminosity-dependent gas-to-dust ratio: $\log \mathrm{M}(\mathrm{HI}) / \mathrm{M}$ (dust) $=3.85+0.3(17.5+\mathrm{M}(\mathrm{B}))$. From this result we may state that at first the cause for irregular dwarfs not being detected by IRAS is due to large gas-to-dust ratios in these galaxies and secondly there is at least no contradiction to the hypothesis of a relation between absolute magnitude and gas-to-dust ratio of dwarf irregular galaxies.

\section{Relation between Gas-to-Dust Ratio and Metallicity}

Since the interstellar dust particles mainly are composed of heavy elements as is demonstrated by the correlation between interstellar extinction and depletion of interstellar gaseous substances exhibiting high condensation temperatures (Field 1974; Jenkins 1989), an observable connection between the gas-to-dust ratio and the metallicity of galaxies should be expected. Indeed, Issa et al. (1990) detected such a relation using data of a small number of spiral galaxies and bright irregulars.

The sample of dwarf irregular galaxies with known gas-to-dust ratio allows the extension of the relation detected by Issa et al. to faint objects. For 15 out of the 46 dwarf irregulars with estimated HI-to-dust ratio the relative abundances of oxygen to hydrogen (by number of atoms), $\log (\mathrm{O} / \mathrm{H})$, which may represent the metallicity are available from the literature. In order to enlarge the sample the eight irregular galaxies brighter than $M(B)=-18.0$ in the catalogue of $S B$ are added.

In spite of a large scatter the plot metallicity, $\log (\mathrm{O} / \mathrm{H})$, versus the gas-to-dust ratio, $\log \mathrm{M}(\mathrm{HI}) / \mathrm{M}$ (dust), shows a clear correlation. The best fit is given by the slope $\mathrm{d} \log (\mathrm{O} / \mathrm{H}) / \mathrm{d} \log \mathrm{M}(\mathrm{HI}) / \mathrm{M}($ dust $)=-0.63 \pm 0.25$, which is in a fair agreement with that following 
from the data by Issa et al. $d \log Z / d \log M(H I) / M(d u s t)=-0.85 \pm 0.10$.

From the observed relation between the gas-to-dust ratio and metallicity, the part of heavy elements in the interstellar medium of irregular galaxies which is condensed in dust particles yields between 4 and 20 per cent.

\section{References}

Field, G.B., 1974. Astrophys. J., 187, 453.

Israel, F.P., 1984. IAU Symp. No. 108, Reidel Publ. Comp., Dordrecht, p. 319.

Issa, M.R., MacLaren, J. and Wolfendale, A.W., 1990. Astron. Astrophys., 236, 237.

Jenkins, E.B., 1989. IAU Symp. No. 135, Kluwer Academic Publishers, Dordrecht, p. 23.

Lequeux, J.M., Peimbert, M., Rayo, J.F., Serrano, A.and Torres-Peimbert, S., 1979. Astron. Astrophys., 80, 155.

Sage, L.J., 1993. Astron. Astrophys., 272, 123.

Schmidt, K.-H. and Boller, T., 1992. Astron. Nachr., 313, 189 (SB).

Schmidt, K.-H., Priebe, A. and Boller, T., 1993. Astron. Nachr., in press.

Skillman, E.D., Kennicut, R.C. and Hodge, P.W., 1989. Astrophys. J., 347, 875.

Young, J.S., Shuding Xie, Kenney, J.D.P. and Rice, W.L., 1989. Astrophys. J. Suppl. Ser., 63, 323. 